\section{Race and Methotrexate Pharmacogenetics in Rheumatoid Arthritis}

\section{To the Editor:}

Fisher and Cronstein are to be commended for their recent metaanalysis on methylenetetrahydrofolate reductase (MTHFR) polymorphisms and methotrexate (MTX) toxicity in rheumatoid arthritis (RA) ${ }^{1}$. Their work exposes the lack of sound pharmacogenetic data on MTX in RA and underscores that there is a long way to go before MTX pharmacogenetics is ready to guide clinical decision-making in RA.

In the discussion, the authors note the paucity of data in the literature to determine whether the presence or absence of a single-nucleotide polymorphism (SNP) has an effect on time to adverse events from MTX. We have reported that the presence of $A B C C 2$ IVS $23+56 \mathrm{~T}>\mathrm{C}$, an intronic $\mathrm{SNP}$ in one of the ABC transporter genes, correlated with time to MTX discontinuation and/or dose decrease due to toxicity in Caucasian patients with RA $(\mathrm{p}<0.0001)^{2}$. Although this is not a MTHFR SNP, to our knowledge, this is the only study to date to demonstrate such a genotype to time to MTX discontinuation association.

The authors also point out that none of the studies included in the analysis provided details on the racial background of the participants and emphasize the importance of race in influencing pharmacogenetics in different populations. Indeed, the SNP frequencies in several of the key genes in MTX pharmacogenetics including MTHFR differ significantly by race $^{3,4}$. Further, we have shown that genotype-toxicity associations vary by race. In our cohort of Caucasians and African Americans with RA, we found that the MTHFR $677 \mathrm{C}>\mathrm{T}$ SNP was associated with MTX toxicity in African Americans with RA but not in Caucasians, and the intronic $A B C C 2$ SNP with time to MTX discontinuation in Caucasians as described above, but not in African Americans with $\mathrm{RA}^{2}$. Others have demonstrated the MTHFR $677 \mathrm{C}>$ T SNP to be associated with MTX toxicity in Caucasians and African Americans with RA ${ }^{4}$. These results supplement those of Fisher and Cronstein's metaanalysis, which showed the MTHFR $677 \mathrm{C}>\mathrm{T}$ SNP to be associated with MTX toxicity in studies that examined populations of Caucasians and Asians, but did not include African Americans. We concede that the sample sizes of these studies were small, a problem all too com- mon with almost all the studies related to MTX pharmacogenetics in RA.

Fisher and Cronstein's metaanalysis once again demonstrates the futility of small-scale pharmacogenetic studies in providing meaningful results that can be applied to individualize patient therapy in clinical practice. Collaborative, multicenter, adequately powered studies, stratified by race, are needed to clarify the muddled state that exists in MTX pharmacogenetics today.

PRABHA RANGANATHAN, MD, MS, Division of Rheumatology, Washington University School of Medicine, 660 S. Euclid Avenue, Campus Box 8045, St. Louis, Missouri 63110; HOWARD McLEOD, PharmD, UNC Institute for Pharmacogenomics and Individualized Therapy, University of North Carolina, Chapel Hill, North Carolina, USA. Address correspondence to Dr. Ranganathan. E-mail: prangana@dom.wustl.edu

\section{REFERENCES}

1. Fisher MC, Cronstein BN. Metaanalysis of methylenetetrahydrofolate reductase (MTHFR) polymorphisms affecting methotrexate toxicity. J Rheumatol 2009;36:539-45.

2. Ranganathan P, Culverhouse R, Marsh S, Mody A, Scott-Horton TJ, Brasington R, et al. Methotrexate pathway gene polymorphisms and their effects on methotrexate toxicity in Caucasian and African American patients with rheumatoid arthritis. J Rheumatol 2008;35:572-9.

3. Ranganathan $P$, Culverhouse R, Marsh S, Ahluwalia R, Shannon WD, Eisen S, et al. Single nucleotide polymorphism profiling across the methotrexate pathway in normal subjects and patients with rheumatoid arthritis. Pharmacogenomics 2004;5:559-69.

4. Hughes LB, Beasley TM, Patel H, Tiwari HK, Morgan SL, Baggott $\mathrm{JE}$, et al. Racial or ethnic differences in allele frequencies of single nucleotide polymorphisms in the methylenetetrahydrofolate reductase gene and their influence on response to methotrexate in rheumatoid arthritis. Ann Rheum Dis 2006;65:1213-8.

J Rheumatol 2010;37:5; doi:10.3899/jrheum.091095 